\title{
AVALIAÇÃO DA TOXICIDADE DE PIRACLOSTROBIN, EPOXICONAZOL E SUA MISTURA EM ALGA Pseudokirchneriella subcapitata
}

\author{
ELIZABETH BIAGIONI PRESTES* \\ CLAUDIO MARTÍN JONSSON** \\ VERA LÚCIA S. S. DE CASTRO***
}

\begin{abstract}
Estudou-se o efeito toxicológico (inibição de crescimento) de formulações fungicidas à base de piraclostrobin e epoxiconazol, isoladamente e em formulação conjugada, sobre a alga Pseudokirchneriella subcapitata mediante determinação da Concentração Efetiva Média $\left(\mathrm{CE}_{50}-72 \mathrm{~h}\right)$ dos princípios ativos de cada uma das formulações. Foram encontrados valores de $\mathrm{CE}_{50}-$ $72 \mathrm{~h}$ de $5,57 \mathrm{mg} . \mathrm{L}^{-1}$ para a formulação à base de piraclostrobin, de $1,14 \mathrm{mg} \cdot \mathrm{L}^{-1}$ para a formulação à base de epoxiconazol e de $0,20 \mathrm{mg}$. $\mathrm{L}^{-1}$ para a formulação conjugada. O piraclostrobin apresentou menor toxicidade às algas em relação aos dados encontrados na literatura, o epoxiconazol resultados semelhantes e a mistura maior toxicidade. A análise isobolográfica e a determinação do Índice de Aditividade (IA) sugerem forte sinergismo da mistura quanto à sua ação tóxica sobre o organismo-teste. Esse fato realça a necessidade de revisão dos padrões limitantes desses fungicidas em corpos de água, os quais tendem a subestimar os efeitos adversos resultantes da combinação dos agentes tóxicos.
\end{abstract}

* Bióloga, Laboratório de Ecotoxicologia e Biossegurança, Embrapa Meio Ambiente, Jaguariúna, SP, Brasil (e-mail: bbiagioni@gmail.com).

** Farmacêutico, Laboratório de Ecotoxicologia e Biossegurança, Embrapa Meio Ambiente, Jaguariúna, SP, Brasil (e-mail: jonsson@cnpma.embrapa.br).

*** Médica Veterinária, Laboratório de Ecotoxicologia e Biossegurança, Embrapa Meio Ambiente, Jaguariúna, SP, Brasil (e-mail: castro@cnpma.embrapa.br). 


\section{INTRODUÇÃO}

Entre os fungicidas disponíveis no mercado destacam-se as misturas de princípios ativos pertencentes às classes dos triazois e estrobilurinas, tais como o epoxiconazol e o piraclostrobin, respectivamente. No mercado brasileiro encontram-se disponíveis para venda formulações à base das duas substâncias isoladas e conjugadas entre si.

A ação dos triazois decorre da inibição do esterol 14a-demetilase (codificado pelo gene CYP51) em bolores e leveduras, bloqueando a biossíntese do ergosterol, componente essencial das membranas celulares desses organismos (ZARN, BRUSCHWEILER e SCHLATTER, 2003). Já as estrobilurinas agem inibindo a respiração mitocondrial pela ligação ao citocromo $b$, bloqueando $a$ geração de ATP (BARTLETT et al., 2002).

Pelas características inerentes de sua utilização, fungicidas podem atingir os ambientes aquáticos circundantes aos locais de aplicação, contaminando águas superficiais e levando injúria à fauna e à flora aquática presentes. Apesar de serem encontrados na literatura trabalhos relativos aos efeitos ecotoxicológicos dos triazois sobre organismos aquáticos, são poucos os estudos sobre as estrobilurinas ou das duas substâncias conjugadas.

A Pseudokirchneriella subcapitata, alga clorofícea unicelular, é amplamente empregada na avaliação de risco e monitoramento de poluentes, sendo recomendado o seu uso por órgãos internacionais (OECD, 1984; GHERARDI-GOLDSTEIN et al., 1990; JANSSEN e HEIJERICK, 2003).

No presente trabalho avaliou-se o efeito toxicológico de formulações fungicidas à base de piraclostrobin, epoxiconazol e sua mistura sobre a alga Pseudokirchneriella subcapitata, mediante determinação da Concentração Efetiva Média $\left(\mathrm{CE}_{50}-72 \mathrm{~h}\right)$ de cada uma das formulações.

\section{MATERIAL E MÉTODOS}

Foram testadas formulações de fungicidas à base de piraclostrobin $(25,0 \% \mathrm{~m} / \mathrm{v})$, epoxiconazol $(12,5 \% \mathrm{~m} / \mathrm{v})$ e de sua mistura (piraclostrobin a $13,3 \% \mathrm{~m} / \mathrm{v}$ e epoxiconazol a $5,0 \% \mathrm{~m} / \mathrm{v}$ ), oriundos de apresentações comerciais disponíveis no mercado. Como organismoteste utilizou-se a alga clorofícea unicelular Pseudokirchneriella subcapitata (anteriormente denominada Selenastrum capricornutum), cultivada em meio conforme metodologia preconizada pela OECD (1984), em sala climatizada sob temperatura controlada a $20 \pm 2^{\circ} \mathrm{C}$ e luminosidade de 1.000 lux. A suspensão algácea foi distribuída em placas de Petri de maneira que no volume final de $15 \mathrm{~mL}$ por placa houvesse a concentração algácea de $10^{4}$ a $10^{5}$ células. $\mathrm{mL}^{-1}$. Adicionaramse os fungicidas a serem testados de maneira a serem obtidas concentrações de 0,$01 ; 0,10$; 1,00; 10,00 e 100,00 mg. L $^{-1}$ de substância ativa. Nos tempos 0, 24, 48 e 72 horas de exposição aos fungicidas foram tomadas alíquotas das suspensões algáceas para a contagem celular, em câmara de Neubauer, a fim de se determinar a Concentração Efetiva Média $\left(\mathrm{CE}_{50}-72 \mathrm{~h}\right)$ dos agentes tóxicos.

O cálculo da $\mathrm{CE}_{50}-72 \mathrm{~h}$ baseou-se na inibição relativa da taxa de crescimento dos organismos em função da concentração do princípio ativo. Para o cálculo das taxas de crescimento utilizaram-se os valores de inclinação da regressão linear das respectivas curvas de crescimento em função do tempo. Os dados foram tratados pelo módulo Simple Regression, contido no programa Statgraphics Plus (MANUGISTICS, 2001), o que permitiu calcular a $\mathrm{CE}_{50}-72 \mathrm{~h}$ e seu intervalo de confiança $95 \%$, além de determinar o modelo de regressão pelo melhor ajuste dos dados.

A análise isobolográfica e o cálculo do Índice de Aditividade, segundo Marking (1977, 1985), permitiram avaliar a ocorrência de efeito sinérgico, antagônico ou aditivo dos ingredientes na mistura sobre o organismo-teste. O grau de magnificação do efeito foi determinado segundo procedimento descrito pelo mesmo autor. 


\section{RESULTADOS E DISCUSSÃO}

\subsection{PIRACLOSTROBIN}

Nos grupos tratados com o fungicida piraclostrobin, verificou-se redução no crescimento da população algácea proporcional ao aumento da concentração de exposição ao agente químico.

$\mathrm{Em} 72$ horas de exposição, considerando-se os valores absolutos, o grupo tratado com a menor concentração do fungicida apresentou a maior população algácea $\left(97,5 \times 10^{5} \mathrm{cél}^{\mathrm{m}} \mathrm{mL}^{-1}\right)$ e o grupo com a maior concentração do fungicida evidenciou a menor população $\left(9,3 \times 10^{5} \mathrm{cél}^{\mathrm{l}} \cdot \mathrm{mL}^{-1}\right)$. 0 grupo controle, com população de $15 \times 10^{5} \mathrm{cé} . \mathrm{mL}^{-1}$ no tempo zero, atingiu contagem maior que sete vezes no último dia de análise $\left(113,8 \times 10^{5} \mathrm{cél} \cdot \mathrm{mL}^{-1}\right)$, como observado na Figura 1.

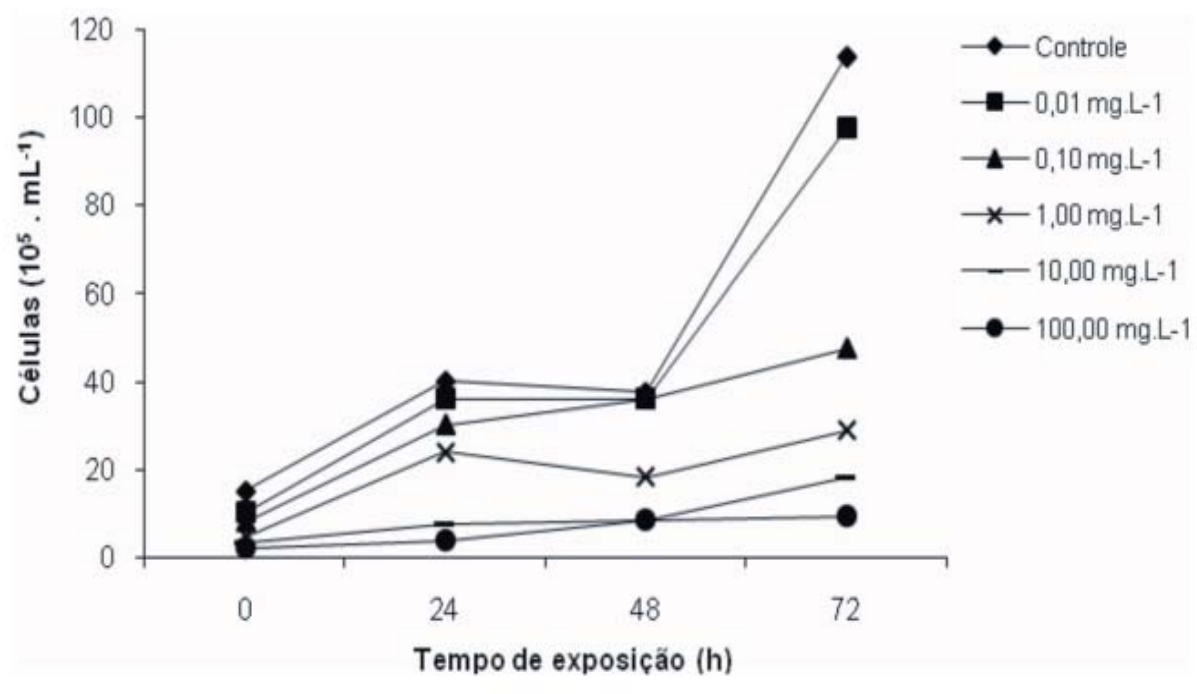

\section{FIGURA 1 - CURVAS DE CRESCIMENTO DE P. subcapitata EXPOSTA A DIFERENTES CONCENTRAÇÕES DE PIRACLOSTROBIN}

Nota: Cada ponto representa a média de observações pertencentes a quatro réplicas.

Avaliando a diferença de crescimento algáceo entre os grupos no tempo de $72 \mathrm{~h}$ de exposição ( $\Delta$ crescimento), observa-se que o grupo exposto a $0,01 \mathrm{mg} \cdot \mathrm{L}^{-1}$ foi o que apresentou o maior crescimento, com aumento total de $87,5 \times 10^{5} \mathrm{cé} \cdot \mathrm{mL}^{-1}$. Já o grupo exposto a $100,00 \mathrm{mg} \cdot \mathrm{L}^{-1}$ revelou o menor aumento total, com 7,3 $\times 10^{5} \mathrm{cél}^{\mathrm{mL}} \mathrm{mL}^{-1}$.

Face aos resultados obtidos, a $\mathrm{CE}_{50}-72 \mathrm{~h}$ para o piraclostrobin foi de $5,57 \mathrm{mg} \cdot \mathrm{L}^{-1}$ (Tabela 1). Ochoa-Acuña et al. (2009) obtiveram valor de $\mathrm{CE}_{50}(72 \mathrm{~h})$ equivalente a $1,40 \mathrm{mg} \cdot \mathrm{L}^{-1}$ para a mesma espécie de alga.

São encontrados na literatura resultados díspares entre trabalhos com fungicidas da classe das estrobilurinas. Ochoa-Acuña et al. (2009), estudando o azoxistrobin com a mesma espécie de alga, constataram $\mathrm{CE}_{50}-72 \mathrm{~h}$ de $0,23 \mathrm{mg} \cdot \mathrm{L}^{-1}$, enquanto Tomlin (2000) estabeleceu o valor de $0,12 \mathrm{mg} \cdot \mathrm{L}^{-1}$. Estudos de toxicidade em algas com outros fungicidas pertencentes à classe das estrobilurinas foram realizados. Godwin et al. (2000) verificaram $\mathrm{CE}_{50}-72 \mathrm{~h}$ de $0,06 \mathrm{mg} \cdot \mathrm{L}^{-1}$ para piraclostrobin. Já para o cresoxim-metílico, estudos divulgados pela Autoridade Nacional de Registro de Químicos Agrícolas e Veterinários da Austrália (NRA, 2000) estabelece $C_{50}-72$ h para $P$. subcapitata de 0,07 mg.L-1. Ochoa-Acuña et al. (2009), estudando a mesma espécie de alga, obtiveram o valor de $\mathrm{CE}_{50}-72 \mathrm{~h}$ de $0,12 \mathrm{mg} \cdot \mathrm{L}^{-1}$ para o trifloxistrobin, outro fungicida da classe das estrobilurinas. 
TABELA 1 - PARÂMETROS TOXICOLÓGICOS PARA P. subcapitata EXPOSTA A

DIFERENTES CONCENTRAÇÕES DE PIRACLOSTROBIN, E

POXICONAZOL E SUA MISTURA POR 72 HORAS

\begin{tabular}{ccccc}
\hline Fungicida & $\begin{array}{c}\mathbf{C E}_{50}-\mathbf{7 2} \mathbf{h} \\
\left(\mathbf{m g} \cdot \mathbf{L}^{-1}\right)\end{array}$ & $\begin{array}{c}\text { Intervalo de } \\
\text { confiança } \\
\left(\mathbf{m g . L}^{-1}\right)\end{array}$ & Modelo de regressão & $\mathbf{R}^{\mathbf{2}}$ (\%) \\
\hline Piraclostrobin & 5,57 & $4,47-7,10$ & $y=\frac{1}{1}(1,02876+0,184662 x)$ & 88,90 \\
\hline Epoxiconazol & 1,14 & $0,90-1,42$ & $y=\exp (0,00882409-0,610256 x)$ & 93,57 \\
\hline $\begin{array}{c}\text { Mistura } \\
\text { (Piraclostrobin } \\
+ \text { Epoxiconazol) }\end{array}$ & 0,20 & $0,14-0,34$ & $y=1,01544-1,12315 \cdot \sqrt{x}$ & 81,73 \\
\hline
\end{tabular}

\subsection{EPOXICONAZOL}

Nos grupos tratados com o epoxiconazol, o aumento da concentração de exposição ao agente químico implicou na redução do crescimento da população algácea. Às 72 horas de exposição, verificou-se a maior população $\left(50,5 \times 10^{5} \mathrm{cél}^{\mathrm{mL}} \mathrm{mL}^{-1}\right)$ em valores absolutos no grupo exposto a $0,01 \mathrm{mg} \cdot \mathrm{L}^{-1}$ e a menor população $\left(2,8 \times 10^{5} \mathrm{cé} . \mathrm{mL}^{-1}\right)$ nos grupos expostos a 10,00 e $100,00 \mathrm{mg} \cdot \mathrm{L}^{-1}$. O grupo controle revelou a maior população ao final do experimento $\left(98,0 \times 10^{5} \mathrm{cé}^{\mathrm{l}} \mathrm{m \textrm {m } ^ { - 1 }}\right)$ e a maior taxa de crescimento populacional, conforme a Figura 2.

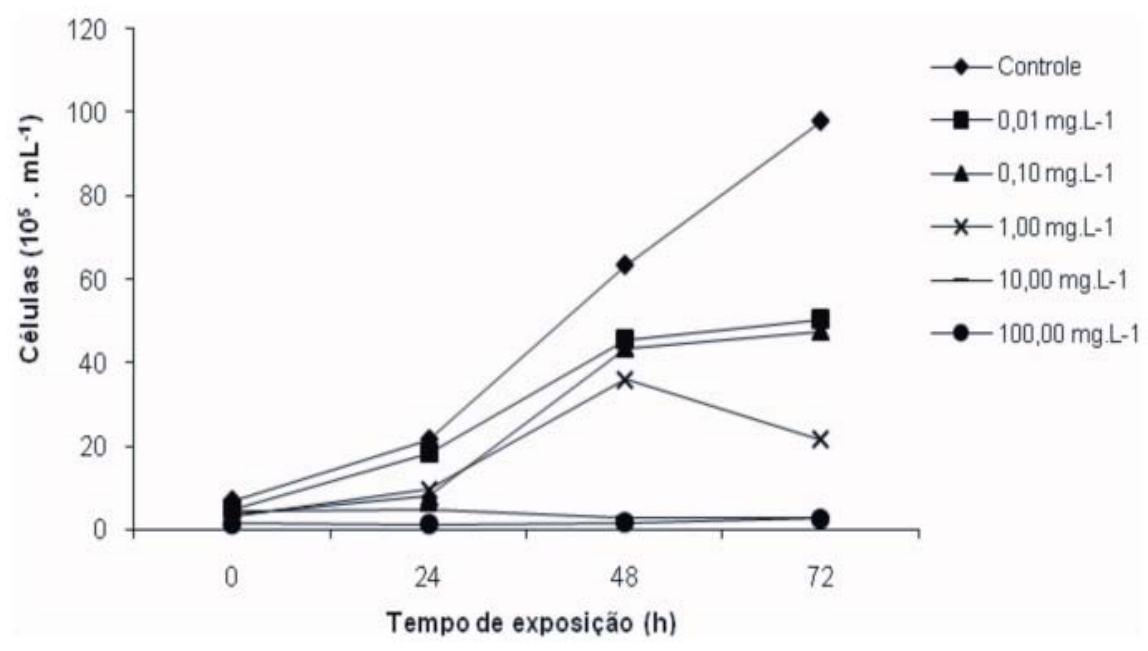

FIGURA 2 - CURVAS DE CRESCIMENTO DE P. subcapitata EXPOSTA A DIFERENTES CONCENTRAÇÕES DE EPOXICONAZOL

Nota: Cada ponto representa a média de observações pertencentes a quatro réplicas.

Face aos resultados obtidos, a $\mathrm{CE}_{50}-72 \mathrm{~h}$ para o epoxiconazol foi de $1,14 \mathrm{mg} \cdot \mathrm{L}^{-1}$ (Tabela 1). O valor encontrado no presente estudo ficou próximo do valor determinado por Probst et al. (2005) em algas verdes, ou seja, $\mathrm{CE}_{50}-72 \mathrm{~h}$ de $1,66 \mathrm{mg} \cdot \mathrm{L}^{-1}$. Ochoa-Acuña et al. (2009) expondo a mesma espécie de alga ao propiconazol encontraram $\mathrm{CE}_{50}-72 \mathrm{~h}$ de $0,39 \mathrm{mg} \cdot \mathrm{L}^{-1}$ para esse fungicida, também pertencente ao grupo dos triazois. 


\subsection{MISTURA (PIRACLOSTROBIN + EPOXICONAZOL)}

Assim como para os produtos testados isoladamente, o aumento da concentração dos componentes da mistura (piraclostrobin + epoxiconazol) exerceu redução no crescimento da população algácea.

Considerando os valores absolutos, o grupo tratado com a menor concentração de fungicida apresentou em 72 horas de exposição a maior população algácea $\left(29,5 \times 10^{5}\right.$ cél. $\left.\mathrm{mL}^{-1}\right)$ e o grupo com a maior concentração, a menor população $\left(3,5 \times 10^{5} \mathrm{cé}^{\mathrm{l}} \cdot \mathrm{mL}^{-1}\right)$. $\mathrm{O}$ grupo controle, com população de $10,5 \times 10^{5}$ cél. $\mathrm{mL}^{-1}$ no tempo zero, demonstrou no último dia de análise aumento próximo de oito vezes na sua população $\left(83,8 \times 10^{5}\right.$ cél.mL-1), conforme observado na Figura 3.

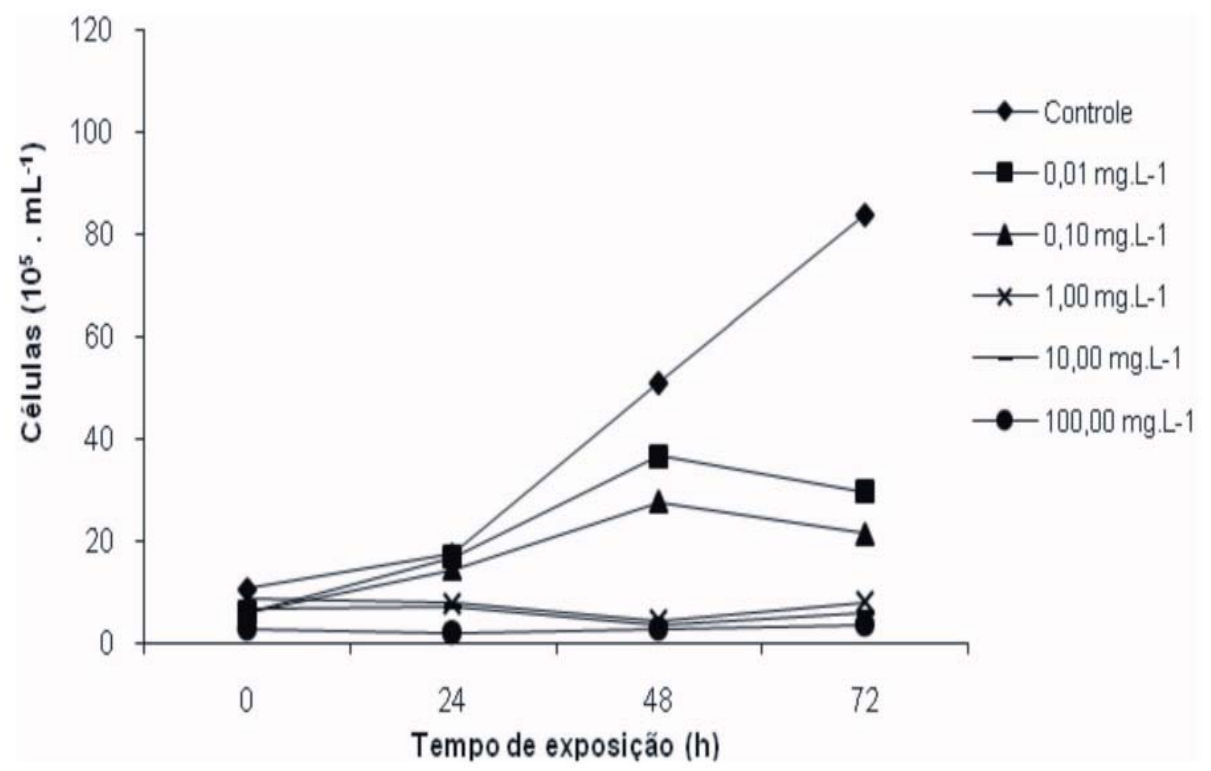

\section{FIGURA 3 - CURVAS DE CRESCIMENTO DE P. subcapitata EXPOSTA A DIFERENTES CONCENTRAÇÕES DA MISTURA (PIRACLOSTROBIN + EPOXICONAZOL)}

Nota: Cada ponto representa a média de observações pertencentes a quatro réplicas.

Ao se analisar a diferença de crescimento algáceo em $72 \mathrm{~h}$ ( $\Delta$ crescimento) observa-se que o grupo exposto a $0,01 \mathrm{mg} \cdot \mathrm{L}^{-1}$ mostrou a maior taxa de crescimento, com aumento total de $23,5 \times 10^{5}$ cél. $\mathrm{mL}^{-1}$. Já os grupos expostos a concentrações iguais ou acima de $1,0 \mathrm{mg} \cdot \mathrm{L}^{-1}$ da mistura apresentaram crescimento irrelevante da sua população.

Face aos resultados obtidos, a $\mathrm{CE}_{50}-72 \mathrm{~h}$ para a mistura (piraclostrobin + epoxiconazol) foi de $0,20 \mathrm{mg} \cdot \mathrm{L}^{-1}$ (Tabela 1 ).

A escassez de trabalhos disponíveis na literatura com a utilização da mistura dificulta a comparação dos resultados. $O$ fabricante de uma das apresentações comerciais, cuja formulação constitui-se de piraclostrobin $(12,5 \% \mathrm{~m} / \mathrm{m})+$ epoxiconazol $(4,7 \% \mathrm{~m} / \mathrm{m})$ divulgou $\mathrm{CE}_{50}-72 \mathrm{~h}>$ $3,00 \mathrm{mg} \cdot \mathrm{L}^{-1}$ para algas, não sendo citada a espécie (BASF, 2009). Para outro produto comercial do mesmo fabricante, com a associação de epoxiconazol $(11,5 \% \mathrm{~m} / \mathrm{m})$ e a estrobilurina cresoximmetílico $(11,5 \% \mathrm{~m} / \mathrm{m})$ consta $\mathrm{CE}_{50}-72 \mathrm{~h}$ para P. subcapitata de $0,39 \mathrm{mg} \cdot \mathrm{LL}^{-1}$ (BASF, 2003).Para a mesma associação em diferentes porcentagens $(12,5 \% \mathrm{~m} / \mathrm{v}$ de epoxiconazol e $12,5 \% \mathrm{~m} / \mathrm{v}$ de cresoxim-metílico), outro fabricante informou $\mathrm{CE}_{50}-72 \mathrm{~h}$ de $0,05 \mathrm{mg} \cdot \mathrm{L}^{-1}$ (MILENIA AGROCIÊNCIAS, 2007).

Considerando os valores de $\mathrm{CE}_{50}-72 \mathrm{~h}$ encontrados neste trabalho, todos os fungicidas 
analisados classificam-se como "altamente tóxicos" conforme critério da U.S. Environmental Protection Agency (1985).

\subsection{ANÁLISE ISOBOLOGRÁFICA}

A análise isobolográfica e o cálculo do Índice de Aditividade (IA) equivalendo a 12,6 sugerem forte sinergismo dos fungicidas na mistura, quanto à sua ação tóxica sobre o organismo-teste. Deste modo, estima-se fator de magnificação de toxicidade de 13,6 vezes em relação à toxicidade individual de cada composto. Esses achados, de certo modo, corroboram as observações de Coors e Frische (2011), que realizaram a predição da toxicidade aquática de formulações comerciais contendo misturas de agrotóxicos. Tais autores detectaram interação sinérgica que impede o crescimento algáceo por misturas de fungicidas que inibem diferentes enzimas da síntese do ergosterol. Além da presença em fungos, esse composto tem sido detectado em algas clorofíceas unicelulares como o maior constituinte dos esterois (PATTERSON, 1969).

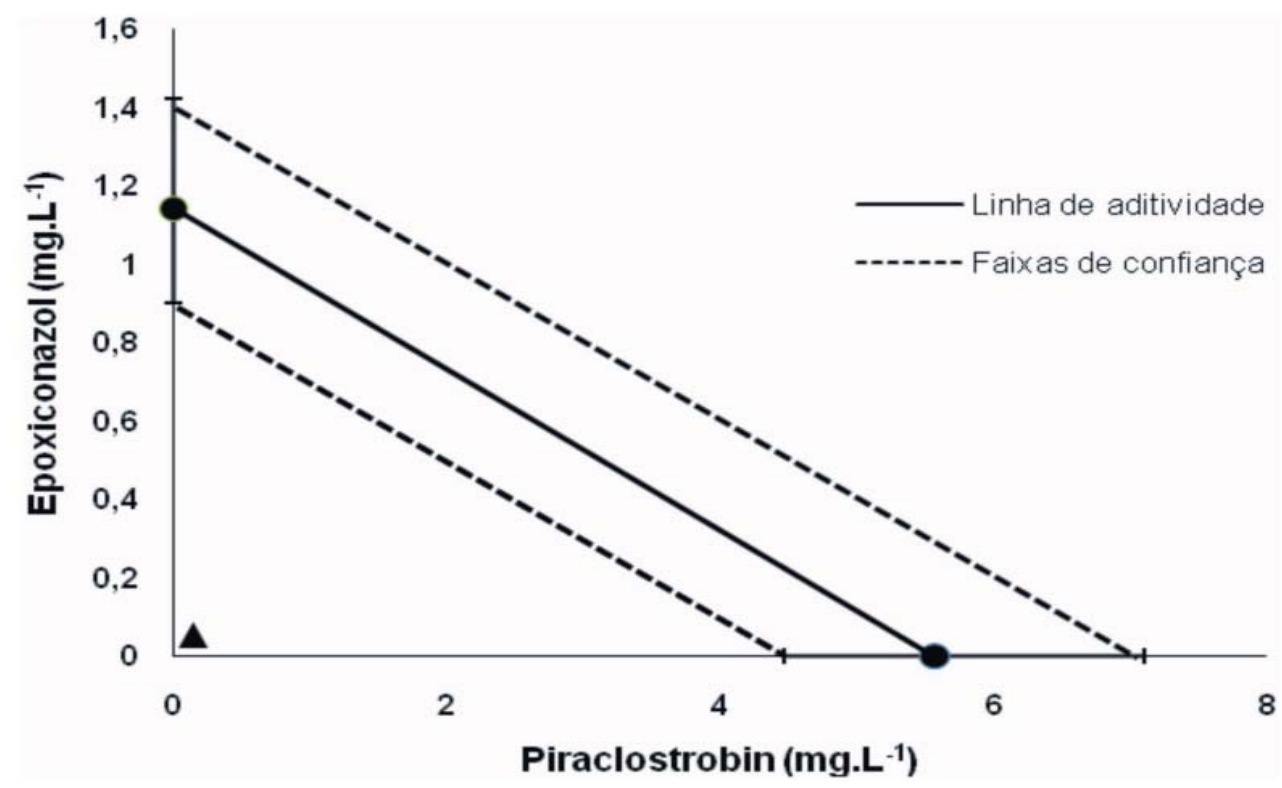

FIGURA 4 - ANÁLISE ISOBOLOGRÁFICA PARA PIRACLOSTROBIN E EPOXICONAZOL

Nota: O ponto abaixo da reta (sinergismo) representa a concentração da combinação piraclostrobin + epoxiconazol que causa $50 \%$ de inibição de crescimento algáceo. O Índice de Aditividade (IA) calculado para esse ponto equivale a 12,6.

\section{CONCLUSÃO}

No presente trabalho, o piraclostrobin apresentou menor toxicidade às algas que os resultados encontrados na literatura. O epoxiconazol, por sua vez, obteve resultado semelhante ao da literatura e a mistura dos dois fungicidas mostrou toxicidade maior. Entretanto, deve-se levar em conta a grande variabilidade dos resultados entre os diferentes trabalhos divulgados na literatura sobre a toxicidade desses produtos em algas.

Estudos posteriores que avaliem a variação de sensibilidade entre os diversos cultivos algáceos, provenientes de laboratórios diferentes poderiam oferecer visão mais ampla sobre a influência desse fator nos testes de toxicidade.

O forte sinergismo entre os dois compostos da mistura realça a necessidade de revisão dos padrões limitantes desses fungicidas em corpos de água, que tendem a subestimar os efeitos adversos resultantes da combinação dos agentes tóxicos. 
Além do fornecimento de informações associadas às estimativas de limites máximos permissíveis na água para misturas de fungicidas, os dados são úteis para o planejamento de estudos em longo prazo, à comparação de toxicidade entre poluentes e o conhecimento de interações de poluentes em organismos não alvo.

\section{ABSTRACT \\ TOXICITY OF FORMULATIONS BASED ON PIRACLOSTROBIN, EPOXICONAZOLE AND ITS COMBINATION ON ALGAE Pseudokirchneriella subcapitata}

Was investigated the toxicological effect (growth inhibition) of fungicides formulations based on piraclostrobin, epoxiconazole and its combined formulation over algae Pseudokirchneriella subcapitata by determination of Mean Effective Concentration $\left(\mathrm{EC}_{50}-72 \mathrm{~h}\right.$ ) of each formulation active compounds. Were observed values of $\mathrm{EC}_{50^{-}}$ $72 \mathrm{~h}$ of $5.57 \mathrm{mg} . \mathrm{L}^{-1}$ for piraclostrobin-based formulation, of $1.14{\mathrm{mg} . \mathrm{L}^{-1}}^{-1}$ for epoxiconazol-based formulation and $0.20 \mathrm{mg}^{-\mathrm{L}^{-1}}$ for combined formulation. Piraclostrobin presented lower toxicity to algae when compared to data exposed on the literature, epoxiconazole had similar results and combined fungicides had a higher toxicity. Isobolographic analysis and additive index (AI) determination suggest a strong synergism of the mixture considering toxic action on test-organism. This fact highlights that is required a revision of those pesticides limiting standard in aquatic environment which tends to underestimate adverse effects resulting from combination of toxic agents.

KEY-WORDS: PIRACLOSTROBIN; EPOXICONAZOLE; TOXICITY; Pseudokirchneriella subcapitata.

\section{REFERÊNCIAS}

1 BARTLETT D. W.; ClOUgh J. M.; GODWIN J. R.; HALL, A. A.; HAMER, M.; PARR-DOBRZANSKI, B. Review: the strobilurin fungicides. Pest Management Science, West Sussex, v. 58, n. 7, p. 649-662, 2002.

2 BASF NEW ZEALAND LIMITED. Allegro ${ }^{\circledR}$. Safety Data Sheet. Auckland, 2003. (Doc.: NZ 6262).

3 BASF CROP PROTECTION. Opera ${ }^{\circledR}$. Cheadle Cheshire, 2009. (Doc.: xmam1249027715.indd).

4 COORS, A.; FRISCHE, T. Predicting the aquatic toxicity of commercial pesticides mixtures. Environmental Sciences Europe, Berlin, v. 23, n. 22, p.1-18, 2011.

5 Gherardi-goldstein, E.; Bertoletti, E.; ZAgAtTo, P. A.; ARAujo, R. P .A.; RAMOS, M. L. L. C. Procedimentos para a utilização de testes de toxicidade no controle de efluentes líquidos. São Paulo: CETESB, 1990. $17 \mathrm{p}$

6 GODWIN, J. R.; BARTLETT, D. W.; CLOUGH, J. M.; GODFREY, C. R. A.; HARRISON, E. G.; MAUND, S. J. Picoxystrobin: a new strobilurin fungicide for use on cereals. In: BCPC CONFERENCE - PESTS AND DISEASES, 2., 2000, Brighton . Proceedings... Brighton: BCPC, 2000. p. $533-540$.

7 JANSSEN, C. R.; HEIJERICK, D.G. Algal toxicity tests for environmental risk assessments of metals. Reviews of Environmental Contamination and Toxicology, New York, v. 178, p. 23-52, 2003.

8 MANUGISTICS Statgraphics Plus: version 5.1 for Windows. Rockville, 2001.

9 MARKING, L. L. Method for assessing additive toxicity of chemical mixtures. In: MAYER, F. L.; HAMELINK, J. L. (Org.). Aquatic toxicology and hazard evaluation. Philadelphia: American Society for Testing and Materials, 1977. p. 99-18.

10 MARKING, L. L. Toxicity of chemical mixtures. In: RAND, G. M.; PETROCELLI, S. R. (Org.). Fundamentals of aquatic toxicology: methods and application. Washington: Hemisphere, 1985. p.164-176.

11 MILENIA AGROCIÊNCIAS S. A. Guapo ${ }^{\circledR}$. Ficha de informações e segurança de produtos químicos. Londrina, 2007. (Revisão de documento, 1).

12 NRA. National Registration Authority for Agricultural and Veterinary Chemicals. Evaluation of the new active kresoximmethyl in the product Stroby WG fungicide. Canberra, 2000.

13 OECD. Organization for Economic Co-operation and Development. Guidelines for the testing of chemicals: 201 - Alga, growth inhibition test. Paris, 1984.

14 OCHOA-ACUÑA, H. G.; BIALKOWSKI, W.; YALE, G.; HAHN, L. Toxicity of soybean rust fungicides to freshwater algae and Daphnia magna. Ecotoxicology, London, v. 18, n. 4, p. 440-446, 2009.

15 PATTERSON, G. W. Sterols of Chlorella - III. Species containing ergosterol. Comparative Biochemistry and Physiology, 
New York, v. 31, n, 3, p. 391-394, 1969.

16 PROBST, M.; BERENZEN, N.; LENTZEN-GODDING, A.; SCHULZ, R. Scenario-based simulation of runoff-related pesticide entries into small streams on a landscape level. Ecotoxicology and Environmental Safety, New York, v. 62, n. 2, p. 145-159, 2005.

17 TOMLIN, C. D. S. (Org.). The pesticide manual. $12^{\text {th }}$ ed. Farnham: British Crop Protection Council, 2000. 1250 p.

18 U.S. Environmental Protection Agency. Hazard Evaluation Division. Standard evaluation procedure: acute toxicity test for freshwater invertebrates. Washington, D.C., 1985.

19 ZARN, J.A.; BRUSCHWEILER, B.J.; SCHLATTER, J.R. Azole fungicides affect mammalian steroidgenesis by inhibiting sterol 14 alfa demethylase and aromatase. Environmental Health Perspective, Durham, v. 111, n. 3, p. 255-261, 2003.

\section{AGRADECIMENTOS}

Os autores agradecem ao Conselho Nacional de Desenvolvimento Científico e Tecnológico (CNPq) e à Fundação de Amparo à Pesquisa do Estado de São Paulo (FAPESP) pelo suporte financeiro ao estudo. 